\title{
Muzio Clementis didaktische Werke: die Klavierschule Op. 42 und der Gradus ad Parnassum Op. 44
}

\section{Muzio Clementi's Pedagogical Works: the Introduction to the Art of Playing on the Pianoforte Op. 42 and the Gradus ad Parnassum Op. 44}

Rossella Marisi / rossellamarisi@hotmail.it

Accademia di Belle Arti, Bologna, IT

\begin{abstract}
This study focuses on two pedagogical works by Muzio Clementi: the Introduction to the Art of Playing on the Pianoforte Op. 42 (1801), addressed to beginners, and the Gradus ad Parnassum Op. 44 (1817-1826), addressed to advanced students. Utilizing Clementi's biography as a point of departure, this study reports that he contributed to innovations in piano pedagogy and improvements in piano-building, and highlights his ability to create a virtuous circle between his careers as a concert performer, a pedagogue, and a piano producer. Thanks to his multifaceted professional expertise, he became one of the most renowned musicians and entrepreneurs in London and gained celebrity and success all over Europe.

Clementi's works are analyzed from a piano-pedagogy point of view, stressing the links between them and other works by the same composer, aimed at being performed in piano recitals, above all the Piano Sonatas. Clementi's pedagogical works are also compared with the ones by some pedagogues of the 18th and 19th centuries: Marpurg, C. Ph. E. Bach, Bemetzrieder, Fenaroli, Türk, Vierling, Dussek and Pleyel, Adam, Pollini, de Montgeroult, Hummel, Kalkbrenner, and Czerny.

A specific sub-section focuses on the everlasting success of Clementi's works, which are still included in Conservatory syllabi, performed in recitals, and included in CDs: this shows that they continue to contribute to the development of a widespread "pianistic culture" among the audience.
\end{abstract}

\section{Key words}

Clementi, Introduction to the Art of Playing on the Pianoforte Op. 42, Gradus ad Parnassum Op. 44, piano pedagogy, 18th and 19th centuries, Kalkbrenner, Czerny 
Viele Wissenschaftler sind sich einig, dass zwischen einer Gesellschaft und ihrer Kultur eine enge Beziehung besteht. ${ }^{1}$ Die Gesellschaft setzt ihre Prioritäten, auf die die Kultur aufgerufen ist zu antworten, und die Kultur zeigt der Gesellschaft, aus der sie selbst entspringt, neue Entwicklungswege. Ins Spiel tritt also ein komplexes Netz von Beziehungen zwischen wirtschaftlichen, ideologischen, politischen, psycho-soziologischen, künstlerischen, und wissenschaftlich-technischen Faktoren, die verursachen, dass jegliche Veränderung an einem der Knoten des Netzes, Auswirkungen auf alle anderen ausübt.

Es ist daher interessant, die Art und Weise zu untersuchen, in der einige didaktische Werke von Clementi die Absichten ihres Autors bezüglich Komposition, Lehre, Konzerttätigkeit und Unternehmertum verwirklichen. Dank seiner vielfältigen Tätigkeit, war Clementi zwar in der Lage Trends zu erfassen, die erst später eintraten, und die andere Musiker und Unternehmer dann zur Vollendung brachten.

\section{Muzio Clementi: Das Leben}

Muzio Clementi wurde am 23. Januar 1752, in Rom, als erstes der sieben Kinder von Nicolò und Magdalena Kaiser, geboren. Da der kleine Muzio sehr früh musikalische Begabung zeigte, ließ ihn sein Vater unter der Leitung von berühmten Meistern, wie Buroni, Cordicelli, Carpini, studieren. Der junge Clementi machte so schnell Fortschritte, dass er schon als vierzehnjähriger die Stelle des Orgelspielers in der Kirche von San Lorenzo in Damaso erhielt.

Zwischen 1766 und Anfang 1767, entschied Sir Peter Beckford auf seiner Reise durch Italien, den sehr jungen Musiker mit sich nach England zu nehmen. Muzio blieb im Dorset bis 1774 zu Diensten von Beckford und nachdem er seine siebenjährige Verpflichtung zu Beckford erfüllt hatte zog Clementi nach London, wo er bald als Tastaturinstrumentalist, Arrangeur und Kopist, ${ }^{2}$ Cembalomeister und sehr wahrscheinlich als Lehrer ${ }^{3}$ bekannt und geschätzt wurde. Zwischen 1780 und 1785 begab sich Clementi wegen Konzert-Touren oft ins Ausland: er war in Paris, Straßburg, ${ }^{4}$ Berlin, Bordeaux, Wien (wo im Jahr 1781 vor Kaiser Josef II ein musikalisches Wettspiel mit Mozart stattfand $)^{5}$ und Bern.

1 Vgl. WEBER, Max. L'etica protestante e lo spirito del capitalismo. Firenze: Sansoni, 1970; HORKHEIMER, Max - ADORNO, Theodor Wiesengrund. Dialettica dell'illuminismo. Torino: Einaudi, 1966; HABERMAS, Jürgen. Storia e critica dell'opinione pubblica. Bari: Laterza, 1971; DURKHEIM, Émile. Rappresentazioni individuali e rappresentazioni collettive. In Le regole del metodo sociologico. Milano: Comunità, 1979; PARK, Robert E. - BURGESS, Ernest W. - MCKENZIE, Roderisk D. La città. Milano: Comunità, 1967; PARSONS, Talcott. Il sistema sociale. Milano: Comunità, 1951; ROSENGREN, Karl E. (Hrsg.). Effects and Beyond: Culture, Socialisation and Lifestyles. London: Routledge, 1994; weitere wichtige Beiträge zu diesem Thema spendeten Antonio Gramsci, Alberto Abruzzese, Italo Lana, Nino Malara.

2 Quarterly Musical Magazine and Review, II, 1820, S. 308-316.

3 PLANTINGA, Leon. Clementi: his life and music. London - New York - Toronto: Oxford University Press, 1977, S. 37-38.

4 UNGER, Max. Muzio Clementis Leben. Langensalza: Hermann Beyer und Söhne, 1914, S. 25.

5 ANDERSON, Emily (Hrsg.). The Letters of Mozart and his Family. London: Palgrave Macmillan, 1989, S. 789-790. 
Nachdem Clementi 1785 endgültig nach London zurückgekehrt war, wurde er zum Komponist und ersten Darsteller der Hanover Square Grand Professional Concerts ernannt, ${ }^{6}$ aber 1796 gab er seiner Karriere als Komponist und Lehrer den Vorrang und schränkte seine öffentlichen Auftritte als Konzertmeister erheblich ein. ${ }^{7}$

In der Tat war Clementi bereits im Jahr 1791 als Lehrer sehr gefragt: dies ist auch durch die Tatsache belegt, dass die Königin von Großbritannien und Irland, Charlotte von Mecklenburg-Strelitz, ihn als Musiklehrer für die Prinzessinnen Augusta und Elizabeth rief. Clementi hatte viele talentierte Studenten. $\mathrm{Zu}$ den berühmtesten gehöhrten Johann Baptist Cramer, Benoît-Auguste Bertini, John Field, Hélène de Nervo de Montgeroult; bemerkenswert waren aber auch der Organist Arthur Thomas Corfe, Maria Parke, Tochter des Oboisten John und Enkelin des Komponisten William Parke, ${ }^{8}$ der Komponist Johann Georg Graeff, ${ }^{9}$ und Theresa Jansen Bartholozzi. ${ }^{10}$

Gegen Ende der neunziger Jahre begann Clementi seine berufliche Laufbahn als Unternehmer und gründete eine Gesellschaft zuerst mit Welcker und Blundell vom gleichnamigen Verlagshaus ${ }^{11}$ und später mit den Klavierherstellern Longman \& Broderip. Während die anderen Gesellschafter wechselten, ${ }^{12}$ blieb Clementi bis zu seiner Pensionierung im Jahr 1830 der Dreh- und Angelpunkt dieses Unternehmens.

Er interessierte sich aktiv an der Firma indem er zu den technischen Verbesserungen der Instrumente beitrug ${ }^{13}$ und machte viele Reisen in ganz Europa, bei denen er seine Klaviere vorstellte und Komponisten kontaktierte um Verlagsverträge zu unterzeichnen. In der Tat waren die Reisen von Clementi, in der Zeit zwischen 1802 und 1810, fast ausschließlich kommerzieller Natur. Er reiste nach Paris, Wien, St. Petersburg, Berlin, Dresden, Prag, Zürich, Leipzig, Riga, Rom und Mailand. ${ }^{14}$ Clementi gab weiterhin auch außerhalb von England Klavierunterricht; zu seinen Studenten dieser Jahre zählten Karl Zeuner, Alexander Klengel ${ }^{15}$ und Ludwig Berger. ${ }^{16}$

6 Morning Herald, 27. Januar 1786.

7 PLANTINGA, Leon. Clementi: his life and music, op. cit., S. 151.

8 WOLLENBERG, Susan - MCVEIGH, Simon (Hrsg.). Concert Life in Eighteenth-century Britain. Aldershot

- Burlington: Ashgate, 2004, S. 284-285.

9 PLANTINGA, Leon. Clementi: his life and music, op. cit., S. 151.

10 BROUGHTON, Vernon Delves (Hrsg.). Court and Private Life in the Time of Queen Charlotte: Being the Journals of Mrs Papendiek, Assistant Keeper of the Wardrobe and Reader to Her Majesty, 2 Bd. London, 1887, I, S. 202-203.

11 PLANTINGA, Leon. Clementi: his life and music, op. cit., S. 155.

12 Bei der Klavierherstellung zählten zu den Geschäftspartnern von Clementi im Laufe der Jahre auch Banger, Hyde, Collard und Davis. Vgl. HUMPHRIES, Charles - SMITH, William C. Music Publishing in the British Isles, from the Beginning until the Middle Part of the Nineteenth Century. New York: Barnes \& Noble, 1970, S. $107-108$.

13 UNGER, op. cit., S. 102-103.

14 PLANTINGA, Leon. Clementi: his life and music, op. cit., S. 191-192.

15 Ibid., S. 208-209.

16 RELLSTAB, Ludwig. Ludwig Berger, ein Denkmal. Berlin: [s. n.], 1846, S. 30. 
Die Jahre 1810-1828 waren für Clementi wirtschaftlich sehr erfolgreich. Seine Klavierfirma wuchs, ${ }^{17}$ im Jahr 1814 wurde er zum Mitglied der Royal Academy of Music von Stockholm ${ }^{18}$ ernannt, seine Symphonien, die in den Jahren zuvor, weit weniger Begeisterung erwecken konnten als jene von Haydn, wurden jetzt in vielen Konzertreihen aufgeführt ${ }^{19}$ und sehr vom Publikum geschätzt, ${ }^{20}$ und sein monumentales Werk Gradus ad Parnassum, das gleichzeitig in Paris, Leipzig und London veröffentlicht wurde, erzielte beachtlichen Erfolg. ${ }^{21}$ Nach einem sehr arbeitsreichen, zeitweise sehr frenetischen Leben, verkaufte Clementi 1830 sein Anteil an der Klavierproduktionsfirma und zog sich auf das Land in den Ruhestand zurück. Zuerst lebte er in Lichfield und später in Evesham, wo er am 10. März 1832 starb.

\section{Das musikalische Leben in London zwischen dem 18. und 19. Jahrhundert}

"Ein echtes Peru für Musiker", so bezeichnete der preußische Historiker und Publizist Johann Wilhelm von Archenholz (1742-1812) London im Jahr 1791. ${ }^{22}$

Mit diesem Satz, wollte Archenholz sicher andeuten, dass London durch seine blühende Wirtschaft und wegen des dort weit verbreiteten Interesses an Theater und Musik, den Musikern ein breites und vielfältiges Angebot an Arbeitsmöglichkeiten zu bieten hatte.

London hatte das damals weltweit beste und gleichzeitig auch das breiteste Angebot an öffentlichen Musikaufführungen.

Das Interesse an der Musik regte die Nachfrage an öffentlichen Musikaufführungen an und damit den Musikinstrumentebau und das Musikverlagswesen. Das Publikum, das die öffentlichen Konzerte besuchte, sorgte auch für eine wachsende Nachfrage an Musikunterricht, ${ }^{23}$ wodurch sich reiche didaktische Aufgaben für die Musiker eröffneten. Die wachsende Zahl an Musikschülern veranlasste die Hersteller, mehr Musikinstrumente zu produzieren, wobei gleichzeitig deren technischen Eigenschaften verbessert wurden, und gab den Verlegern den Anreiz, immer mehr musikalische Werke zu veröffentlichen, sowohl Erstveröffentlichungen von Kompositionen zeitgenössischer

17 PLANTINGA, Leon. Clementi: his life and music, op. cit., S. 227.

18 Ibid., S. 234.

19 Gazetteer, 24. Februar 1787.

20 Morning Chronicle, 8. März 1824.

21 PLANTINGA, Leon. Clementi: his life and music, op. cit., S. 245.

22 ARCHENHOLZ, Johann Wilhelm von. A Picture of England. Dublin: P. Byrne, 1791, S. 122.

23 Vor allem bei Mädchen aus guter Familie, verfolgte die instrumentale Praxis oft verschiedene Ziele: sie erforderte den Einsatz an Disziplin, gab Gelegenheit zur Gefühlsäußerung, und Anlass zum Glänzen an den gesellschaftlichen Abenden. Letzteres erhöhte die Möglichkeit, eine gute Heirat zu machen'. Vgl. APPLETON, Elizabeth. Private Education, or A Practical Plan for the Studies of Young Ladies. London: Henry Colburn, 1816, S. 189; COLMAN, George. The Musical Lady. A Farce. London: T. Becket und P. A. Dehondt, 1762, S. 6. 
Musiker, als auch Neuauflagen von klassischen Werken. Einige ${ }^{24}$ der veröffentlichten Werke dienten zur Unterhaltung der Musikliebhaber, ${ }^{25}$ andere, die ein spezifisch didaktisches Ziel hatte, waren für Schüler bestimmt, ob sie nun Berufsmusiker werden wollten oder nicht, ${ }^{26}$ und weitere Werke waren dazu bestimmt, in das Konzertrepertoire von Solisten ${ }^{27}$ und professionellen Ensembles aufgenommen zu werden.

\section{Die Klavierschule für Anfänger...}

Im Jahr 1801 veröffentlichte Clementi mit der Opusnummer 42, die Introduction to the Art of Playing on the Pianoforte, eine in drei Teile gegliederte Klavierschule für Anfänger, die jeweils in zwei Abschnitte unterteilt sind. Der erste Teil ist eine Zusammenfassung der Notenschrift und der wesentlichen Argumente der Theorie, dazu gehört auch die Einstufung der Intervalle, die Zeichen der Abkürzung, Anweisungen zu Tempo, Verzierungen, und Schlüssel. Der zweite Teil gibt Anweisungen zur Stellung der Hand, zum Fingersatz bei den Tonleitern, zum Fingerwechsel auf der gleichen Taste, und zeigt technische Übungen für kurze Passagen, Tonleitern, Arpeggios, und Passagen in Terzen und Sexten; ${ }^{28}$ der dritte Teil enthält mehrere kurze Stücke verschiedener Autoren.

Der theoretische Teil ist wahrscheinlich der weniger eigenständige. Plantinga findet eine starke Ähnlichkeit dieses Teils der Klavierschule von Clementi mit der Klavierschule von Dussek, Introduction to the Art of Playing the Piano Forte or Harpsichord, im Jahr 1796 veröffentlicht, und deutet die Möglichkeit an, dass Clementi mehr als nur einen Anstoß von dieser genommen hatte. ${ }^{29}$ Es ist jedoch anzumerken, dass praktisch alle Klavierschulen für Anfänger aus dieser Zeit die Erklärung der Theorie auf eine sehr ähnlichen

24 Die zur Unterhaltung der Musikliebhaber vorgesehenen Musikstücke waren trotz ihrer Verbreitung nicht immer von hoher, künstlerischer Qualität: zu den sehr erfolgreichen, aber nicht unbedingt von großem musikalischem Wert, gehörten verschiedene Kompositionen von Joseph Wölfl und das im Jahr 1788 komponierte Stück Die Schlacht von Prag von Franz Koczwara. Vgl. HOHL TRILLINI, Regula. The Gaze of the Listener. English Representations of Domestic Music-Making. Amsterdam - New York: Rodopi, 2008, S. 64-65.

25 Anfang 1800 wurde in der Regel angenommen, dass die Kompetenz der weiblichen Mitglieder des englischen Mittelstandes, die zum Vergnügen Tasteninstrumente spielten, so hoch war, dass diese zur exquisite execution fähig wären und damit alle anderen Amateurmusikspieler in Europa überträfen. Vgl. BURGH, Allatson. Anecdotes of Music, Historical and Biographical, in a Series of Letters from a Gentleman to his Daughter. London: Longman, Hurst, Rees, Orme and Brown, 1814, S. VII.

26 Vgl. GELLRICH, Martin. Üben mit Lis(z)t. Wiederentdeckte Geheimnisse aus der Werkstatt der Klaviervirtuosen. Frauenfeld: Waldgut, 1992.

27 Beispiele zu den Repertoires der Solisten in der genannten Zeit findet man unter anderem in GRATTAN FLOOD, William Henry. John Field of Dublin, Inventor of the Nocturne. Dublin: Martin Lester, 1921, S. 5-6.

28 Es mag überraschen, dass Anfängern technische Übungen mit Terzen und Sexten bestimmt sind, die in der Regel für fortgeschrittene Studenten als geeignet angesehen werden. Es ist jedoch zu beachten, dass Clementi in seinen Kompositionen für alle Lehrstufen sehr oft Passagen mit Terzen anwendet und er selbst ein Virtuose dieser Technik war, was sogar sein "Gegner" Mozart anerkannte. Vgl. ANDERSON, Emily (Hrsg.). The Letters of Mozart and his Family, op. cit., S. 850. Das deutet darum darauf hin, dass Clementi überzeugt war die brillante Ausführung dieser Passagen sei wesentlich für Pianisten, und deren Aneignung nicht besonders schwer.

29 PLANTINGA, Leon. Clementi: his life and music, op. cit., S. 166. 
Weise beginnen und darum kann man davon ausgehen, dass der Ansatz von Clementi ein bewusster Bezug auf eine bereits etablierte Tradition der Lehre ist und kein Ergebnis eines Plagiats.

Die Neuheit von Clementi ist jedoch die Entscheidung, im dritten Teil der Klavierschule eine reiche Sammlung an Werken von Autoren aus verschiedensten Ländern zu veröffentlichen, ${ }^{30}$ sowohl alten (wie zum Beispiel J.S. Bach, ${ }^{31}$ Händel, D. Scarlatti, F. Couperin und Rameau) als auch zeitgenössischen (wie Haydn, Mozart, Plevel, Cramer, Dussek, Steibelt, Field und Beethoven). ${ }^{32}$ Neben Werken, die eigens für Tasteninstrumente komponiert wurden, sind Charakterstücke enthalten, die der damals modernen Vorliebe für exotische Klänge ${ }^{33}$ verpflichtet sind, sowie Arien aus aktuellen Opern und Oratorien. ${ }^{34}$

Clementi trug mit diesem Werk aktiv zu einem damals höchst wichtigen wirtschaftlich-künstlerischen Kreislauf bei: Viele Zuhörer, die den öffentlich zugänglichen Musikaufführungen beiwohnten, erhöhten die Nachfrage an Musikunterricht, und durch diesen wurde das Interesse geweckt wie gefestigt, noch mehr Opern, Oratorien und Instrumentalkonzerte zu geniessen.

Die Klavierschule von Clementi, welcher der Autor in der Ausgabe von 1811 einen Appendix von Präludien und Übungen hinzufügte, war in Europa weit verbreitet. Es gab zahlreiche, gedruckte Ausgaben in England und im Ausland. ${ }^{35}$ Selbst Beethoven schätzte diese Klavierschule sehr. ${ }^{36}$ Er empfahl seinem Neffen, Clementis Schule zu folgen, um

30 In der Tat, bereits die zwei Teile des Werks Dialog über die richtige Weise Orgel und Instrumente zu spielen, von Girolamo Mancini, Diruta genannt, geschrieben und veröffentlicht 1593 beziehungsweise 1609, enthalten eine breite Sammlung von Werken für Orgel von verschiedenen Autoren: Diruta selbst, Merulo, die zwei Gabrieli, Luzzaschi, Romanini, Quagliati, Bell'Haver, Guami, Mortaro, Fattorini und Banchieri (Vgl. DIRUTA, Girolamo. Il Transilvano. Dialogo sopra il vero modo di sonar organi et istromenti da penna. Venezia: [s. n.], 1593, anastatischer Druck, Bologna: Forni, 1997.). Allerdings fallen wichtige Unterschiede sofort auf. Diruta widmet seine Arbeit Musikern in Ausbildung (und nicht einem breiten Publikum zu dem auch Liebhaber gehören können) und fügt in die Anthologie Stücke von zeitgenössischen Komponisten ein, die auch seine Landsleute waren. Es fehlen darum zwei der interessantesten Elemente die die Anthologie von Clementi kennzeichnen: das synchronisch-diachronische Panorama und der Leitgedanke, durch das Lehrwerk eine richtige Industrie der Musik und Unterhaltung ins Leben zu rufen.

31 Clementi kaufte aus der Sammlung Beckford das Londoner Autograph des Wohltemperierten Claviers und benutzte es als Quelle für einige Stücke die er in den zweiten Teil seiner Klavierschule aufnahm.Vgl. PLANTINGA, Leon. Clementi, Virtuosity, and the German Manner. Journal of the American Musicological Society, XXV, 1972, S. 323-329.

32 Der Ländlerische Tanz von Beethoven, den Clementi in die Lesson XXXV mit dem Titel Waltz einfügte, ist eins der ersten Stücke von Beethoven die in England veröffentlicht wurden. Vgl. PLANTINGA, Leon. Clementi: his life and music, op. cit., S. 165-166.

33 Zwischen diesen spanische, italienische, irische und russische Arien, und Hymnen aus verschiedenen Ländern.

34 Zwischen diesen Arien aus La molinara von Paisiello, Le Barbier de Séville von A. L. Baudron, Saul e Sansone von Händel.

35 LIMONGELLI, Silvia. La didattica per principianti e dilettanti. In RATTALINO, Piero (Hrsg.). Trecento anni, li dimostra? Il pianoforte nella società di oggi. Como: Tipografia Nani Editrice, 2000, S. 123-128.

36 SCHINDLER, Anton Felix. Beethoven as I knew Him. A Biography. Hrsg. D.W. Macardle. Chapel Hill: University of North Carolina Press, 1966, S. 379-380. 
eine gute Musikausbildung zu erwerben. ${ }^{37}$ Er besorgte sich sogar verschiedene Ausgaben dieser Klavierschule. ${ }^{38}$

\section{... und der Gradus ad Parnassum}

Schon seit 1801 interessierte sich Clementi eingehend an der Zusammenstellung einer Klavierschule für fortgeschrittene Studenten, wie er selbst in einem Brief an den Verleger Härtel bezeugt. In diesem erwähnt Clementi seine Absicht, eine gewisse Anzahl seiner Musikstücke für didaktische Zwecke in einem einzigen Korpus zu fassen, dessen Titel zuerst Studium sein sollte. ${ }^{39}$ Später entschied der Komponist, seine Sammlung Gradus ad Parnassum zu nennen, teilweise um an das in 1725 veröffentlichte, gleichnamige Werk von J.J. Fux anzuspielen, und teilweise um sie von der von Cramer zwischen 1804 und 1810 veröffentlichten Sammlung Studium für Klavier, die gleichfalls ein didaktisches Ziel hatte, zu unterscheiden.

Der Gradus ad Parnassum von Clementi ist ein Werk in drei Bänden, die zwischen 1817 und 1826 veröffentlicht wurden ${ }^{40}$ und den Reifeweg und die musikalische Entwicklung bezeugen, die sein Autor in fünfundvierzig Berufsjahren machte. Es unterstreicht die progressiv wachsende Fähigkeit als Instrumentalist, die Entwicklung seiner Ausdrucksweise in der Komposition und die genaue Darlegung und Vertiefung seines Lehrdenkens. ${ }^{41}$

Einige der Stücke des Gradus sind regelrechte Übungen, nicht besonders ehrgeizig vom musikalischen Standpunt aus, die bereits in der Überschrift das vorbestimmte Bildungsziel hervorheben (zum Beispiel, die Unabhängigkeit der Finger zu entwickeln). Viele Etüden sind den typischen Schwierigkeiten gewidmet, die ein Pianist auf sich nehmen und überwinden muß, um die Vollkommenheit zu erreichen, wie zum Beispiel Tonleitern, Arpeggios, Progressionen von Terzen, Sexten und Oktaven, mehrmals wiederholte Noten und Triller. Die technischen Schwierigkeiten, deren Meisterung der Komponist und Lehrer Clementi in seinen Werken verlangte, sind offensichtlich jene, deren Überwindung er als wesentlich zur Ausbildung eines Pianisten ansah und die der Klaviervirtuose Clementi selbst so gut beherrschte, ${ }^{42}$ dass sogar Mozart ihm ein Lob aussprach. ${ }^{43}$

37 MARANO, Maria. Muzio Clementi e la fondazione della didattica pianistica. Cosenza: Luigi Pellegrini, 2007, S. 30 .

38 ANDERSON, Emily (Hrsg.). The Letters of Beethoven. New York: St. Martin's Press Inc., 1961, S. iii, 1250, 1308, 1313 und 1323.

39 UNGER, op. cit., S. 213-214.

40 PLANTINGA, Leon. Clementi: his life and music, op. cit., S. 233, 237 und 245.

41 PADULA, Alessandra. Musica e pedagogia tra rigore scientifico e creatività artistica. La forma dello Studio. In PERIGOZZO, Enrico (Hrsg.). Il Quaderno del Conservatorio, n. 3. [S. 1.]: Conservatorio di Musica di Pescara, 2005, S. 33-35.

42 Vgl. PADUlA, Alessandra. Virtuosismo e virtuosi. In PERIGOZZO, Enrico (Hrsg.). Il Quaderno del Conservatorio, n. 2. [S. 1.]: Conservatorio di Musica di Pescara, 2004, S. 69-71.

43 ANDERSON, Emily (Hrsg.). The Letters of Mozart and his Family, op. cit., S. 789-790. 
Instrument-Virtuosen auszubilden, war nicht das einzige Ziel von Clementi, sehr wahrscheinlich auch nicht sein Hauptziel. Indem er dem Schüler eine große Anzahl an Werken unterbreitete, diese meist von großem, musikalischen Wert, und in verschiedenen Formen strukturiert, ${ }^{44}$ darunter Suiten, Sonatensätze, Scherzi, Kanons, Fugen und Charakterstücke, so verfolgte Clementi das Ziel, den Schüler zu einem richtigen Musiker auszubilden, der mit Ausdruckskraft im Stande ist, den vielfältigen musikalischen Ausdruck eines Stückes zu ergründen. ${ }^{45}$

Einerseits bezeugt der Gradus spieltechnische Prinzipien und Unterrichtsziele Clementis, andererseits zeigt er auch die Entwicklung seiner kompositorischen Fähigkeiten. In der Tat, in mehreren Etüden des Gradus setzt Clementi Motive oder ganze Teile aus zuvor veröffentlichten Kompositionen ein, aber nicht ohne diese zuvor einer tiefen Revision durch Kürzungen, Erweiterungen und harmonische Änderungen verschiedenster Weise, unterzogen zu haben. ${ }^{46}$

Der Gradus bezeugt nicht nur das Leben und die Kunst seines Autors, sondern auch die dominante Rolle die das Klavier auf der europäischen Musikszene des 19. Jahrhunderts einzunehmen begann. ${ }^{47}$ Eine Rolle zu der Clementi selbst auf entscheidender Weise beitrug, wie die ihm auch außerhalb von England verliehenen Anerkennungen beweisen, ${ }^{48}$ sowie der Ehrentitel 'Vater des Klaviers' der ihm von der zeitgenössischen Presse $^{49}$ und von Musikern wie Wesley ${ }^{50}$ und Moscheles ${ }^{51}$ einstimmig beigemessen wurde, und auch auf seiner Grabinschrift in Westminster Abbey geschrieben steht. ${ }^{52}$

\section{Weitere, zeitgenössische Klavierschulen}

Um die Neuheit der Klavierschule von Clementi besser zu unterstreichen, scheint es mir angebracht kurz die wichtigsten Merkmale anderer, zeitgenössischer Klavierschulen hervorzuheben.

Viele Klavierschulen, die zwischen der zweiten Hälfte des 18. Jahrhunderts und der ersten Hälfte des 19. Jahrhunderts veröffentlicht wurden, geben der Behandlung der Grundsätze über die rechte Stimmführung und das Harmonisieren sowie den Prinzipien der Kunst des Begleitens einen großen Platz. Es gehörte in der Tat zu den Aufgaben der

44 Vgl. PADULA, Alessandra. Forma come contenuto, 2 Bd. Torino: Giacomelli, 2002.

45 PADULA, Alessandra. L'interpretazione come percorso di conoscenza: confronti, problemi, prospettive. München - Ravensburg: Grin, 2007, S. 50-51.

46 PLANTINGA, Leon. Clementi: his life and music, op. cit., S. 271-272.

47 WEBER, Max. I fondamenti razionali e sociologici della musica. In WEBER, Max. Economia e società. Milano: Edizioni di Comunità, 1961, Anhang zu Bd. 2, S. 845-849.

48 Unter denen besonders eindrucksvollen war die bereits erwähnte Ernennung zum Mitglied der königlichen Musikakademie von Stockholm. Vgl. PLANTINGA, Leon. Clementi: his life and music, op. cit., S. $233-234$.

49 Quarterly Musical Magazine and Review, X, 1828, S. 86; The Ladies' Penny Gazette, 15. Dezember 1832.

50 Reminiscences of Samuel Wesley, British Museum, Add. MS. 27593, 147.

51 MOSCHELES, Charlotte. Life of Moscheles, 2 Bd. Boston: Adamant Media Corporation, 2001, I, S. 192.

52 PLANTINGA, Leon. Clementi: his life and music, op. cit., S. 249. 
Tasteninstrumentalisten Akkordfolgen in verschiedenen Formen und Figuren umzusetzen, präludieren und Solisten oder Ensemble begleiten zu können.

Mehrere Lehrwerke für Tasteninstrumente widmen darum einen großen Teil dem Aufbau dieser Fähigkeiten aufgrund der Gewissheit, dass diese auch von Anfängern beherrscht werden müssen. Nur Werke die sich an mehr fortgeschrittene Ausführer richten, wie zum Beispiel die von C. Ph. E. Bach und Türk, geben auch der Ausdrucksfähigkeit Platz.

Mit der Zeit festigte sich aber die Überzeugung, dass der Pianist mehr nach seiner Ausführung der Stücke, die eigens für dieses Instrument komponiert wurden, gefordert werde, als mit dem Improvisieren von Präludien oder der Begleitung. ${ }^{53}$ Bei den neuen Klavierschulen werden deshalb immer mehr Fragen von Körperhaltung, Anschlag, Egalität und Fingersatz behandelt, um den Pianisten dazu zu leiten, mit Klangbrillanz und Ausdrucksfähigkeit Stücke zu spielen, die gerade diese Fähigkeiten ins Licht rücken wollten. ${ }^{54}$

\section{Friedrich Wilhelm Marpurg: Die Kunst das Clavier zu Spielen (1750)}

Der deutsche Komponist und Theoretiker Friedrich Wilhelm Marpurg (1718-1795) kannte persönlich wichtige Vertreter der deutschen Aufklärung, wie Winckelmann und Lessing, und französische Intellektuelle wie Voltaire, D’Alembert und Rameau. In 1749/1750 gründete und leitete er eine der ersten Musikzeitungen Der critische Musicus an der Spree. Er schrieb viele Werke der Theorie und Kritik, unter denen das Werk Die Kunst das Clavier zu Spielen, welches zum ersten Mal in Berlin in 1750 veröffentlicht und danach ins Französische übersetzt und mit dem Titel Principes du clavecin 1756 veröffentlicht wurde. Das Werk richtet sein Augenmerk ausschließlich auf die Begleitung und die Grundsätze des Harmonisierens.

Carl Philipp Emanuel Bach: Versuch über die wahre Art das Clavier zu spielen (1753)

Der deutsche Komponist, Cembalist und Orgelspieler Carl Philipp Emanuel Bach war einer der berühmtesten Clavieristen seiner Zeit. Er war am Hof von Friedrich II von Preußen tätig und wurde dann Musikdirektor und Kantor in Hamburg.

Im Vorwort seines Versuch über die wahre Art das Clavier zu spielen, beschreibt C. Ph. E. Bach mit analytischer Klarheit die Fähigkeiten, die ein Klavierspieler haben muß um seinen zahlreichen und verschiedenen Aufgaben recht zu werden. Dazu gehören Fingerfertigkeit, Improvisierungsfähigkeit, um ein vorgegebenes Thema auszuarbeiten, sichere Ausführung und Ausdruck beim prima-vista-Spiel sowie die Fähigkeit einen Generalbass gut harmonisieren zu können.

Im ersten Teil seiner Klavierschule (die 1753 veröffentlicht wurde) konzentriert C. Ph. E. Bach seine Aufmerksamkeit auf die Interpretation. In der Einleitung präzisiert

53 BURNEY, Charles. A General History of Music from the Earliest Ages to the Present Period (1766-1784). London: G. T. Foulis, 1935, S. 997.

54 BUSBY, Thomas. A Dictionary of Music, Theoretical and Practical. To which is prefixed a Familiar Introduction to the First Principles of that Science. London: Sir Charles Phillips \& Co., 1823, S. 214. 
er selbst, dass die richtige Weise, ein Tasteninstrument zu spielen, auf drei miteinander verbundenen Prinzipien basiert: den richtigen Fingersatz, die richtige Ausführung der Verzierungen und die gute Interpretation. In den drei Kapiteln des Werkes werden diese Themen ausgelegt, und die theoretischen Grundlagen werden mit zahlreichen Beispielen illustriert.

\section{Anton Bemetzrieder: Leçons de clavecin, et principes d'harmonie (1771)}

Anton Bemetzrieder (1739-1808) wurde im Elsass geboren, zog aber dann nach Paris, wo er ein Freund des Philosophen Diderot und Cembalolehrer seiner Tochter Angelica wurde. In der Klavierschule von Bemetzrieder, Leçons de clavecin, et principes d'harmonie, 1771 in Paris veröffentlicht, findet man drei Gestalten von denen nur die Rolle bekannt ist (der Lehrer, der Schüler, der Philosoph), hinter denen aber offensichtlich Bemetzrieder und Vater wie Tochter Diderot verborgen sind. Diese Figuren stellen in Dialogform die theoretischen Grundlagen der Musik vor. Die Klavierschule weist dem gründlichen Studium der Harmonielehre und des Improvisierens eine zentrale Bedeutung zu, und zeigt verschiedene Beispiele von harmonischen Progressionen mit figurierter Begleitung.

\section{Fedele Fenaroli: Regole musicali per principianti di cembalo (1775)}

Der Komponist und Lehrer Fedele Fenaroli (1730-1818) wurde in Lanciano in den Abruzzen geboren, war jedoch hauptsächlich in Neapel tätig, wo er begabte Studenten wie Cimarosa und Mercadante hatte. Er trug zur Vereinigung der neapolitanischen Konservatorien bei, aus der das königliche Kollegium der Musik entstand, von dem Fenaroli Direktor wurde.

Das Werk von Fenaroli, Regole musicali per $i$ principianti di cembalo (Regeln der Musik für Cembaloanfänger), 1775 in Neapel veröffentlicht, gehört mehr der Kategorie der Traktate als jener der Klavierschulen an, da es ausschließlich theoretisch ist. Es erklärt sehr detailliert die Praxis der Harmonisierung des Basses und fasst in wenigen Sätzen Ratschläge zur Ausführung zusammen: "Hinsichtlich der Anwendung der Finger [...], dies wird von der lebhaften Stimme des gelernten Meisters gelehrt; verschiedene Passagen erfordern verschiedene Stellungen der Finger [...], und wollte man dies schriftlich erklären, würde man die Gedanken der Anfänger mit langen und langweiligen Erklärungen verwirren; darum werden diese Erklärungen der Weisheit der Lehrer überlassen" “.55

\section{Daniel Gottlob Türk: Clavierschule oder Anweisung zum Clavierspielen für Lehrer und Lernende (1789)}

Der deutsche Theoretiker, Komponist, Cembalist und Organist Daniel Gottlob Türk (1750-1813) war Organist und Kantor, Musikdirektor und dann Professor an der Universität Halle. Sein 1789 veröffentlichtes umfangreiches Werk Clavierschule oder Anweisung zum Clavierspielen für Lehrer und Lernende, besteht aus mehreren Kapiteln, welche die theoretischen Grundlagen, den Fingersatz, die Verzierung und die Grundsätze für eine gute Ausführung beinhalten.

55 FENAROLI, Fedele. Regole musicali per i principianti di cembalo. Napoli: [s. n.], 1775, S. 6. 
Türk betont die Wichtigkeit der Sicherheit, Klarheit, Verständnis der Empfindungen des Stückes und ihrer Wiedergabe, Wahl und Ausführung der Verzierungen. Das Werk, das mehr als vierhundert Seiten umfasst, enthält nur zwölf von Türk komponierte kurze Stücke, die sich an Anfänger wenden.

\section{Johann Gottfried Vierling: Versuch einer Anleitung zum Präludieren (für Ungeübtere) (1794)}

Der deutsche Komponist und Organist Johann Gottfried Vierling (1750-1813) war Schüler von Carl Philip Emanuel Bach und Johann Philipp Kirnberger. Neben Instrumentalmusik und Gesang schrieb er ein Werk über Harmonielehre und Komposition, Allgemein fasslicher Unterricht im Generalbass mit Rücksicht auf dem jetzt herrschenden Geschmack in der Composition durch treffende Beispiele erläutert, welches 1805 veröffentlicht wurde und das Werk Versuch einer Anleitung zum Präludieren (für Ungeübtere), 1794 veröffentlicht. Letzteres richtet sich an Spieler von Tasteninstrumenten und enthält zahlreiche figurierte Bässe; für jeden einzelnen $\mathrm{Ba}$ sind verschiedene Formen des Präludierens aufgezeigt und deren Hauptprinzipien hervorgehoben. Das didaktische Ziel ist es, dem Schüler die verschiedenen Möglichkeiten bewusst zu machen und ihn in die Lage zu versetzen, diese in persönlicher Weise zu kombinieren, so dass der Schüler fließende Geschmeidigkeit und Kreativität erwirbt und damit einen persönlichen Stil erreicht.

\section{Jan Ladislav Dussek und Ignaz Pleyel: Méthode pour le pianoforte (1797)}

Das Werk Méthode pour le pianoforte wurde fast vollständig vom Komponisten und Pianisten Jan Ladislav Dussek (tschechisch auch Dusík, 1760-1812) geschrieben. Er war ein berühmter Klaviervirtuose der in Deutschland, Holland, Russland, Frankreich und England tätig war, während Ignaz Pleyel (1757-1831) sich nur um die Ausgabe kümmerte und kleine Ergänzungen machte. Die Méthode beginnt mit einem wichtigen theoretischen Teil, der Notenschrift, Verzierungen, Klassifizierung der Intervalle behandelt, und Charakter- und Tempoanweisungen gibt. Der zweite Teil widmet sich der Körperhaltung, der Haltung der Hand auf der Klaviatur und den Grundsätzen der Fingerbewegung, des Fingersatzes, und der dynamischen Klanggestaltung. Verschiedene Übungen betreffen kurze Passagen im Umfang von wenigen Tönen (einschließlich Übungen, in denen mehrere Noten gehalten werden und eine Note oft wiederholt wird), Tonleitern, Arpeggios, Terzen, Sexten und Oktaven. Die Méthode enthält nur 24 kleine Musikstücke, die von steigender Schwierigkeit sind, und offensichtlich didaktischen Zwecken dienen.

\section{Louis Adam: Méthode de piano du Conservatoire (1804)}

Der elsässische Pianist, Lehrer und Komponist Louis Adam (1758-1848) war durch 45 Jahre Lehrer am Conservatoire de Paris. Zeuge dieser eindrucksvollen Dozentenzeit ist auch das Werk Méthode de piano, das mit dem Bericht der Kommission beginnt, die mit der Untersuchung dieser Klavierschule beauftragt war, um seine Anwendung im Conservatoire zu entscheiden; zitiert werden in dem Werk auch die Lobesworte, die Bernard Sarrette, Gründer und Präsident des Conservatoires, diesem Opus spendet. Die Méthode setzt sich aus mehreren Teilen zusammen: aus einem theoretischen Teil, 
einem Teil mit Ratschlägen zur Ausführung und einem Teil mit Stücken die didaktischen Zwecken dienen. Der theoretische Teil ist sehr umfangreich und behandelt Notenschrift, Verzierungen und Abkürzungen. Der zweite Teil, der Ratschläge zu einer guten Ausführung beinhaltet, betrifft Legato, Stakkato, Ausführung der Verzierungen und spezifische Übungen zu den nach und nach erläuterten Themen. Die meisten Übungen betreffen die Fingertechnik, Tonleitern und Arpeggios. Der praktische Teil beinhaltet Cinquante leçons progressives doigtées pour le petites Mains, von Adam selbst komponiert und Choix de Morceaux dans le diffèrens Caracteres et Mouvements. Letzteres ist eine Sammlung von Werken von verschiedenen Autoren (wie Johann Sebastian Bach, Carl Philipp Emanuel Bach, Domenico Scarlatti, Händel, Mozart und Clementi) die mit der Angabe des richtigen Fingersatzes vervollständigt sind und damit zur Besserung der Feinmotorik und des Vortragsstils des Schülers beitragen.

\section{Francesco Pollini: Metodo per clavicembalo (1811)}

Francesco Pollini (1762-1846) wurde in Wien angeblich von W. A. Mozart und in Mailand von Nicola Zingarelli ausgebildet. Er bewies sich als vielseitiger Künstler, und war als Schauspieler, Sänger, Komponist, Violinist und Pianist tätig.

Sein Metodo, das auf der Titelseite erklärt, dass es vom „königlichen Konservatorium Mailand angewendet" und auch ,für die Erziehungshäuser im Reich" komponiert wurde, ist in drei Teile gegliedert: der erste Teil betrifft die Tonleitern, die die Grundlage der Fingerfertigkeit bilden; der zweite Teil konzentriert sich auf Stilfragen (darunter die Ausführung der Verzierungen, den Anschlag, den Gebrauch der Pedale); der dritte Teil betrifft Kadenzen und harmonische Wendungen. Jedes Prinzip wird zuerst dargelegt und dann durch musikalische Beispiele erläutert, deren Ziel es ist zu erklären, wie und wann jedes Prinzip angewendet werden soll.

Große Wichtigkeit wird der Unabhängigkeit der Finger und der Gleichmäßigkeit in der Ausführung der Noten mit gleichem Wert gegeben. Um diese Ziele zu erreichen, bietet Pollini viele technische Übungen an, die den richtigen Fingersatz sehr genau angeben und auf den Grundlagen der Klaviertechnik beruhen. Zu diesen gehören das Artikulieren bei stillstehender Hand, ohne Daumenuntersatz und Ausdehnung der Hand, die Tonleitern, die Arpeggios, die Terzen, die Sexten und die Oktaven. Noch interessanter ist wahrscheinlich der Teil, der die Theorie des Anschlags betrifft, und erklärt, wie der Schüler lernen kann, jedes Musikstück mit dem passenden Anschlag zu spielen.

\section{Hélène de Montgeroult: Cours complet pour l'enseignement du forté piano conduisant progressivement des premiers éléments aux plus grandes difficultés (1820)}

Die Cembalistin, Pianistin und Komponistin Hélène de Montgeroult (1764-1836) studierte unter Dussek, Hüllmandel und Clementi und gehörte zu den besten Cembalisten und Pianisten ihrer Zeit. Da sie eine Aristokratin war, wurde sie während der Französischen Revolution zum Tod verurteilt, entging aber der Hinrichtung wegen ihrer hohen musikalischen Begabung: Vor dem Wohlfahrtsausschuß, der dabei war, ihr Todesurteil zu verordnen, improvisierte sie mit künstlerischem Schwung und politischem Scharfsinn Variationen über das Thema der Marseillaise, was ihr das Leben rettete. 1795 war sie 
als erste Frau Klavierdozentin am Conservatoire de Paris, und fing noch im selben Jahr an, Material für ihren 1820 in Paris veröffentlichten Cours complet pour l'enseignement du forté piano: conduisant progressivement des premiers éléments aux plus grandes difficultés zu sammeln. Diese dreibändige Klavierschule enthält 972 Übungen und 114 progressive Etüden. Ziele der Klavierschule sind eher die Fingerfertigkeit und das Gleichgewicht der Hand, als die Stärke der einzelnen Finger und die dadurch erzielende Klangkraft.

Dies könnte jedoch mit der damaligen Verbreitung von medizinischen Studien in Zusammenhang stehen, die die anatomischen und physiologischen Unterschiede zwischen männlichen und weiblichen Musiker betonten. Darum verlangte man von erfolgreichen Pianistinnen jener Zeit schönen Klang, singende Phrasierung und Ausdruckskraft, während man annahm, dass Kraft und virtuose Technik eher Merkmale der männlichen Pianisten wären.

Johann Nepomuk Hummel: Ausführlich theoretisch-practische Anweisung zum PianoforteSpiel vom ersten Elementar-Unterricht an bis zur vollkommensten Ausbildung (1828)

Der österreichische Pianist und Komponist Johann Nepomuk Hummel (1778-1837) war vor allem Schüler Mozarts und Johann Georg Albrechtsbergers, der Überlieferung nach aber auch Salieris und Clementis. Er war mit Goethe, Schiller, Beethoven und Schubert befreundet und ein außergewöhnlicher Virtuose, dessen Kennzeichen Klarheit und Reinheit des Klanges waren.

Seine in drei Volumen unterteilte Klavierschule, geht vom ersten Anfang am Klavier bis zur höchsten Ausbildung. Hummel richtet seine Aufmerksamkeit auf jeden Aspekt der Klaviertechnik, und widmet jedem Gebiet zahlreiche Übungen. Man findet zum Beispiel 155 Übungen ohne Daumenuntersatz und Ausdehnung der Hand, und fast 400 Übungen zur Ausdehnung der Finger und Hand durch Sexten, Septimen und Oktaven, die Hummel als wesentlich bei der Ausbildung des Virtuosen ansah.

Die Klavierschule verbreitete sich sehr schnell in ganz Europa und wurde auch sehr von Liszt geschätzt. ${ }^{56}$

Friedrich Wilhelm Kalkbrenner: Méthode pour apprendre le piano-forte à l'aide du guidemains (1830)

Der deutsche Pianist und Komponist Friedrich Wilhelm Kalkbrenner (1785-1849) war ein herausragender Absolvent des Conservatoire de Paris und erzielte später Ruhm und wirtschaftlichen Erfolg als Virtuose, Lehrer (er war unter den ersten, die Fortbildungskurse schufen) und Partner der Klavierbau-Firma Pleyel \& Co. Zusammen mit Freunden, unter ihnen Méhul und Rossini, trug Kalkbrenner mit einem Darlehen zur finanziellen Sanierung der Firma Pleyel bei, die bald nach 1810 in eine Krise geraten war. 1829 verzichtete er auf die Rückerstattung des Darlehens und entschloß sich stattdessen, Gesellschafter von Ignaz und Camille Pleyel zu werden; deshalb war er ab nun an den Gewinnen der Firma beteiligt, die in den Jahren 1846 und 1847 besonders beträchtlich waren.

56 Vgl. "Hummel und das Fortepiano“, www.hummel-gesellschaft-weimar.de (Zugriff 22.08.2016). 
Seine 1830 veröffentlichte Méthode pour apprendre le piano-forte à l'aide du guide-mains, setzt sich das Ziel, nicht so sehr die Kraft des Unterarms zu erhöhen, sondern eher Kraft und Ausdauer der einzelnen Fingern zu entwickeln; dieses Ziel zu erreichen, betrachtete Kalkbrenner als unentbehrlich für einen Pianisten, der mit Erfolg ein Konzertrepertoire erwerben wollte. Kalkbrenner bezieht sich bewußt auf die Klavierschule von Clementi ${ }^{57}$ und folgt seinem Beispiel indem seine Méthode aus einem eher kurzen theoretischen Teil besteht, einer Reihe von technischen Übungen, die immer schwieriger werden, und einer Sammlung von 24 Übungen und Etüden deren Schwierigkeitsgrad fortlaufend zunimmt.

In der Méthode ist auch das modèle de guide mains dargestellt, ein Gerät das dem Schüler helfen sollte, die richtige Stellung der Hand auf der Tastatur zu erwerben.

\section{Carl Czerny: Vollständige theoretisch-praktische Pianoforte-Schule op. 500 (1839)}

Der Österreicher Carl Czerny (1791-1857), war ein Schüler von Beethoven und ein berühmter Klavierlehrer, bei dem neben vielen anderen Leopoldine Blahetka und Franz Liszt ausgebildet wurden. Er schrieb viele didaktische Werke, unter denen das Opus 500 besonders wichtig ist. Der Komponist definiert darin das Klavier als "König der Instrumente" und bezeugt damit die zentrale Rolle, die dieses Instrument am Anfang des 19. Jahrhunderts zugewiesen wurde. Er empfiehlt das Klavierstudium, auch weil das Klavierspiel Muskeln und Skelett des Spielers keine Probleme verursacht. Czerny betont auch, dass durch die Verbesserungen an der Klaviermechanik der musikalische Geschmack gewachsen ist, der nun eine ähnliche Entwicklung und Verbesserung der Ausführung fordert.

Dieses Ziel setzt sich das Werk Opus 500, das in drei Teile gegliedert ist. Jeder Teil verbindet die theoretischen Konzepte mit der Erklärung der auf die Tonerzeugung ausgerichteten Gesten, und begleitet diesen theoretischen Input mit musikalischen Beispielen, in der Länge von wenigen Takten bis zu einigen Seiten.

Der erste Teil des Werkes betrifft Artikulation, Ausdehnung der Finger und der Hand, Bewegungen der Hand auf der Tastatur, Kreuzungen, Doppelgriffen und Akkorden; der zweite Teil behandelt Tonleitern, technische Übungen und Fingersatz-Beispiele; der dritte Teil beschäftigt sich mit der Entwicklung der musikalischen Ausdrucksfähigkeit, den Erwerb der Fähigkeit, zu transponieren, zu präludieren und auf dem Klavier zu improvisieren, sowie schließlich auch der Technik des Klavierstimmens.

Hauptsächlich im dritten Teil, der besonders der Interpretation gewidmet ist, nennt Czerny Werke von anderen Autoren (C.P.E. Bach, Mozart, Clementi, Beethoven, Dussek, Steibelt, Ries, Kalkbrenner, Field, Moscheles, Hummel), doch hat er diese nicht in seine Klavierschule aufgenommen. Tatsächlich stellt bereits der Untertitel des Werks (,mit allen nötigen, zu diesem Zweck eigens komponierten, zahlreichen Beispielen') klar, dass die in Czernys Klavierschule enthaltenen Stücke alle von ihm selbst komponiert wurden.

57 Kalkbrenner definiert Clementi nähmlich als «mit einem höheren Genie ausgestattet» und kennt ihn als Lehrer der «den von uns verfolgten Weg zeichnete», an. Vgl. KALKBRENNER, Friedrich Wilhelm. Méthode pour apprendre le piano-forte à l'aide du guide-mains. Paris: Meissonnier Fils, 1830, S. 22. 


\section{Der Erfolg der didaktischen Werke von Clementi}

Seit den zwanziger Jahren des 19. Jahrhunderts leisteten zahlreiche bedeutende Pianisten und Lehrer ihren Beitrag zur Blütezeit der auf Etüden und Übungen ausgerichteten Veröffentlichungen. Unter diesen erinnern wir an Moscheles, der in den Jahren 1825/1826 seine 24 Etüden op. 70 veröffentlichte, an Liszt, der 1826, im Alter von nur fünfzehn Jahren, die Études en douze exercises veröffentlichte, an Schumann, der seine Studien für das Pianoforte nach Capricen von Paganini bearbeitet 1832/1833 schrieb, und an Chopin, der 1833 die Etüden op. 10 komponierte. Die Verbreitung der Sammlungen von Etüden, die nicht in Klavierschulen enthalten waren, führte zu einem fortschreitenden Funktionalitätsverlust der Klavierschulen alter Prägung: Die anthologischen Abschnitte der Klavierschulen konnten in der Tat mit der Menge und Vielfalt der Etüden, die nach und nach selbständig veröffentlicht wurden, nicht mehr mithalten. (Nicht zu vergessen ist freilich auch, dass der Begriff „Etude“ auch für Konzertstücke verwendet wurde, die einen virtuosen Vortrag zeigen sollten, aber nicht mehr didaktische Werke waren.) Grosse Erfolge hatten hingegen die Veröffentlichungen jener Lehrwerke, die bereits Etüden-Sammlungen waren, oder die einen Teil enthielten, der separat als Etüden oder Übungen veröffentlicht werden konnte.

Aus diesen Gründen hatte die Introduction to the Art of Playing on the Pianoforte von Clementi anhaltenden Erfolg, hauptsächlich der Appendix, der separat mit dem Titel Préludes et exercises doigtés veröffentlicht wurde. Was den Gradus betrifft, der bereits in Form einer Sammlung von Etüden dargestellt war, gab es viele Nachdrucke, sowohl vom gesamten Werk als auch von Etüden-Auswahlen; ${ }^{58}$ einige von diesen wurden von berühmten Lehrern wie Callcott, Macfarren, ${ }^{59}$ Köhler, ${ }^{60}$ Lebert, ${ }^{61}$

58 Mehreren Wissenschaftlern nach haben diese Auswahlen zu der Verbreitung der Lehrwerke von Clementi beigetragen, aber in einem gewissen Sinn wurde dadurch deren musikalischer Wert verkannt. Die Folge war, dass diese Klavierschulen nicht mehr als ein "pianistisches Mikrokosmos" betrachtet wurden, sondern als einfache Sammlungen von Übungen: damit wurde ihr musikalischer Wert reduziert. Vgl., unter den Anderen, KENTNER, Louis. Piano. New York: Schirmer, 1976, S. 138.

59 Vgl. BAILLIE, Laureen. The catalogue of printed music in the British Library to 1980. London: K. G. Saur, 1982, Bd. 12, S. 282.

60 HOFMEISTER, Adolph. Handbuch der musikalischen Literatur, oder allgemein systematisch geordnetes Verzeichnis der in Deutschland und in den angrenzenden Ländern erschienenen Musikalien, bearbeitet und herausgegeben von Adolph Hofmeister, 5. Band oder 2. Ergänzungsband die von Anfang 1860 bis Ende 1867 neu erschienenen und neu aufgelegten musikalischen Werke enthaltend. Leipzig: [s. n.], 1868, S. 151; HOFMEISTER, Adolph. Handbuch der musikalischen Literatur, oder allgemein systematisch geordnetes Verzeichnis der im deutschen Reiche und in den angrenzenden Ländern erschienenen Musikalien, 7. Band oder 4. Ergänzungsband die von Anfang 1868 bis Ende 1873 neu erschienenen und neu aufgelegten musikalischen Werke enthaltend. Leipzig: [s. n.], 1876, S. 98; HOFMEISTER, Adolph. Handbuch der musikalischen Literatur, oder allgemein systematisch geordnetes Verzeichnis der im deutschen Reiche und in den angrenzenden Ländern erschienenen Musikalien, 8. Band oder 5. Ergänzungsband die von Anfang 1874 bis Ende 1879 neu erschienenen und neu aufgelegten musikalischen Werke enthaltend. Leipzig: [s. n.], 1881, S. 96; HOFMEISTER, Adolph. Handbuch der musikalischen Literatur, oder allgemein systematisch geordnetes Verzeichnis der im deutschen Reiche und in den angrenzenden Ländern erschienenen Musikalien, 9. Band oder 6. Ergänzungsband die von Anfang 1880 bis Ende 1885 neu erschienenen und neu aufgelegten musikalischen Werke enthaltend. Leipzig: [s. n.], 1887, S. 113 .

61 HOFMEISTER, Adolph. Handbuch der musikalischen Literatur, oder allgemein systematisch geordnetes Verzeichnis der in Deutschland und in den angrenzenden Ländern erschienenen Musikalien, bearbeitet und herausgegeben von Adolph Hofmeister, 5. Band oder 2. Ergänzungsband die von Anfang 1860 bis Ende 1867 neu erschienenen und neu 
Tausig, ${ }^{62}$ Kullak, Germer, Riemann, Bischoff, Klindworth, ${ }^{63}$ Cesi,${ }^{64}$ Buonamici, ${ }^{65}$ Mugellini, ${ }^{66}$ Andreoli, ${ }^{67}$ Frugatta ${ }^{68}$ und Sgambati ${ }^{69}$ publiziert.

Einige Ausgaben führen auf ihrer Titelseite die Musikakademien, die Konservatorien und die Musikinstitute an, die die Etüden von Clementi in ihrem Lehrprogramm verwendeten. Zum Beispiel das vom Verleger Lucca veröffentlicht Buch 25 Studi, trägt die Aufschrift „,on der königlichen Akademie von S.ta Cecilia in Rom“ angewendet ${ }^{70}$ und die von Buonamici ausgewählten 50 Studi nennt das Musikinstitut von Florenz ${ }^{71}$ als

aufgelegten musikalischen Werke enthaltend, op. cit., S. 151; HOFMEISTER, Adolph. Handbuch der musikalischen Literatur, oder allgemein systematisch geordnetes Verzeichnis der im deutschen Reiche und in den angrenzenden Ländern erschienenen Musikalien, 8. Band oder 5. Ergänzungsband die von Anfang 1874 bis Ende 1879 neu erschienenen und neu aufgelegten musikalischen Werke enthaltend, op. cit., S. 96; HOFMEISTER, Adolph. Handbuch der musikalischen Literatur, oder allgemein systematisch geordnetes Verzeichnis der im deutschen Reiche und in den angrenzenden Ländern erschienenen Musikalien, 9. Band oder 6. Ergänzungsband die von Anfang 1880 bis Ende 1885 neu erschienenen und neu aufgelegten musikalischen Werke enthaltend, op. cit., S. 113; HOFMEISTER, Adolph. Handbuch der musikalischen Literatur, oder allgemein systematisch geordnetes Verzeichniß der im deutschen Reiche und in den angrenzenden Ländern erschienenen Musikalien, 10. Band oder 7. Ergänzungsband die von Anfang 1886 bis Ende 1891 neu erschienenen und neu aufgelegten musikalischen Werke enthaltend. Leipzig: [s. n.], 1893, S. 124.

62 HOFMEISTER, Adolph. Handbuch der musikalischen Literatur, oder allgemein systematisch geordnetes Verzeichnis der in Deutschland und in den angrenzenden Ländern erschienenen Musikalien, bearbeitet und herausgegeben von Adolph Hofmeister, 5. Band oder 2. Ergänzungsband die von Anfang 1860 bis Ende 1867 neu erschienenen und neu aufgelegten musikalischen Werke enthaltend, op. cit., S. 151.

63 HOFMEISTER, Adolph. Handbuch der musikalischen Literatur, oder allgemein systematisch geordnetes Verzeichniß der im deutschen Reiche und in den angrenzenden Ländern erschienenen Musikalien, 10. Band oder 7. Ergänzungsband die von Anfang 1886 bis Ende 1891 neu erschienenen und neu aufgelegten musikalischen Werke enthaltend, op. cit., S. ohne 124-145; HOFMEISTER, Adolph. Handbuch der musikalischen Literatur, oder allgemein systematisch geordnetes Verzeichnis der im deutschen Reiche, in den Ländern deutschen Sprachgebietes sowie der für den Vertrieb im deutschen Reiche wichtigen, im Auslande erschienenen Musikalien, 11. Band oder 8. Ergänzungsband die von Anfang 1892 bis Ende 1897 neu erschienenen und neu aufgelegten musikalischen Werke enthaltend. Leipzig: [s. n.], 1900, S. 142.

64 CESI, Beniamino. Metodo per lo studio del pianoforte. Milano: Ricordi, 1897.

65 HOFMEISTER, Adolph. Handbuch der musikalischen Literatur, oder allgemein systematisch geordnetes Verzeichniß der im deutschenReiche, in den Ländern deutschen Sprachgebietes sowie der für den Vertrieb im deutschen Reiche wichtigen, im Auslande erschienenen Musikalien, 14. Band oder 11. Ergänzungsband die von Anfang 1909 bis Ende 1913 neu erschienenen und neu aufgelegten musikalischen Werke enthaltend. Leipzig: [s. n.], ohne Jahresangabe, S. 121.

66 HOFMEISTER, Adolph. Handbuch der musikalischen Literatur, oder allgemein systematisch geordnetes Verzeichniß der im deutschen Reiche, in den Ländern deutschen Sprachgebietes sowie der für den Vertrieb im deutschen Reiche wichtigen, im Auslande erschienenen Musikalien, 13. Band oder 10. Ergänzungsband die von Anfang 1904 bis Ende 1908 neu erschienenen und neu aufgelegten musikalischen Werke enthaltend. Leipzig: [s. n.], ohne Jahresangabe, S. 127.

67 ANDREOLI, Carlo. Scelta sistematica e progressiva delle opere di Clementi. Milano: Ricordi, 1878.

68 CLEMENTI, Muzio. Gradus ad Parnassum, 34 studi per pianoforte scelti ed ordinati secondo i programmi uniformi di Licenza e Magistero stabiliti dal Ministero della I.P. per gli Istituti Musicali del Regno. Hrsg. Frugatta. Milano: Ricordi, 1898.

69 CLEMENTI, Muzio. Gradus ad Parnassum, 40 studi scelti. Hrsg. Sgambati. Milano: Ricordi, 1916.

70 CLEMENTI, Muzio. 25 studi estratti dal Gradus ad Parnassum. Milano: Lucca, 1871.

71 CLEMENTI, Muzio. Per le classi di Pianoforte. R. Istituto musicale di Firenze. 50 studi estratti dal Gradus ad Parnassum da far seguito agli studi di Cramer editi da Hans von Bülow. Progressivamente ordinati e provveduti di nuove diteggiature annotazioni e di segni per la esecuzione dei medesimi da Giuseppe Buonamici. Firenze: Genesio Venturini, 1894. 
besonderen Verwendungsort der Ausgabe. All dies bezeugt, dass das von seinen Zeitgenossen gelobte Lehrwerk von Clementi, auch nach seinem Tod weiterhin studiert und geschätzt wurde. ${ }^{72}$

Ein weiterer Beweis der didaktischen und artistischen Vitalität der pianistischen Werke von Clementi ist die Tatsache, dass auch heute zahlreiche seiner Musikstücke während der öffentlichen Klavierprüfungen in den Konservatorien und den Musikhochschulen vorgetragen werden. In der Tat sind diese - um Beispiele aus Italien zu nennen, oft im Ministerialprüfungsprogramm der sogenannten ,alten Studienordnung;, die in ganz Italien gilt, enthalten ${ }^{73}$ sowie auch im Lehrplan und Prüfungsprogramm des akademischen Diploms der 1. oder 2. Ebene, über deren Details jedes Konservatorium und jede Musikhochschule selbst entscheidet. ${ }^{74}$

Es überrascht daher nicht, dass die Klavierstücke von Clementi oft bei öffentlichen musikalischen Abenden vorgeführt werden. ${ }^{75}$ Noch wichtiger ist aber, dass angesehene Interpreten häufig Stücke von Clementi in ihre $\mathrm{CDs}^{76}$ und ihre Konzertprogramme ${ }^{77}$ aufnehmen.

Noch heutzutage nimmt eben das Werk von Muzio Clementi bei der Ausbildung der Pianisten eine wichtige Rolle ein, und trägt durch öffentliche Aufführungen zu der Entwicklung einer im Publikum weitverbreiteten ,Klavierkultur' bei. $^{78}$

72 Vgl. BACHARACH, Alfred Louis - PEARCE, John R. (Hrsg.). The musical companion. San Diego - New York - London: Harcourt Brace Jovanovich, 1984, S. 606.

73 Vgl. Königliche Verordnung R. D. 11. 12. 1930, n. 1945 in MISTRETTA, Pietro (Hrsg.). L'insegnamento musicale in Italia. Firenze: Le Monnier, 1986, S. 149-150.

74 Vgl. zum Beispiel die Programme des Konservatoriums Florenz unter http://www.consfi.it/oldwebsite/ index.php-id=547.html (Zugriff 23. 08. 2016); die Programme des Konservatoriums Fermo unter http://www. conservatorio.net/cms/testi.php?id_rub=129369930489062\&id_testo=129372286117187(Zugriff 23. 08. 2016); die Programme des Konservatoriums Rom http://www.conservatoriosantacecilia.it/offerta-didattica/ vecchio-ordinamento-2/ (Zugriff 23. 08. 2016).

75 Vgl. zum Beispiel Secondo appuntamento con i saggi dei giovani musicisti del Venezze http://www. rovigooggi.it/articolo/2010-06-10/secondo-appuntamento-con-i-saggi-dei-giovani-musicisti-del-venezze/\#.V7S6dph8u00 (Zugriff 23. 08. 2016 ); programma dei saggi - Conservatorio di Monopoli Nino Rota http://diazilla. com/download/963782 (Zugriff 23. 08. 2016).

76 Unter anderen erinnern wir an die Aufnahmen von L. Alvini. Muzio Clementi: A Portrait. Frame, $2002 ;$ A. Bacchetti et al. Muzio Clementi: Gradus ad Parnassum op. 44. Arts Music, 2004; M. Campanella et al. Clementi: 23 studi obbligatori dal "Gradus ad Parnassum". Warne, 1995; W. Horowitz. A Baroque E Classical Recital. Sony Classical, 1994; D. Laval. Clementi: Six suites du Gradus ad Parnassum. Accord, 1996; P. Spada. Muzio Clementi: Piano Works, vol. 18. Arts, 1999; V. Vitale et al. Muzio Clementi: Gradus ad Parnassum. Incisione integrale dei 100 studi, 5 LP, Fonit Cetra, 1981.

77 Unter anderen, erwähnen wir das am 12.03.2005 in Ravello von Paolo Scanabissi gehaltenes Konzert, http://guide.supereva.it/musica_classica/interventi/2005/03/200234.shtml (Zugriff 23. 08. 2016); das am 21. 05. 2016 in Bergamo von Ronald Brautigam gehaltenes Konzert http://www.festivalpianistico.it/tag/concerti-a-bergamo-festival-2016/ (Zugriff 23. 08. 2016); das am 03. 06. 2016 in Bergamo von Daniele Fasani und Filippo Gorini gehaltenes Konzert,http://www.festivalpianistico.it/progetto-clementi-il-3-giugno-si-recupera-il-concerto-di-gorini-e-fasani/ (Zugriff 23. 08. 2016); das am 16.04.2011 in Rom von Laura Ruzza gehaltenes Konzert, http://www.funweek.it/roma/eventi/concerti-roma-gratis-settimana-cultura-programma.php; das am 22. 07. 2014 in Roana von Giovanni Nesi gehaltenes Konzert,http://www.asiago.it/it/eventi/art_artemusica_cultura-concerto_del_pianista_giovanni_nesi/ (Zugriff 23. 08. 2016).

78 MARISI, Rossella. Musica e divulgazione culturale. München - Ravensburg: Grin, 2009, S. 4 und 11. 


\section{Bibliography}

ANDERSON, Emily (Hrsg.). The Letters of Beethoven. New York: St. Martin's Press Inc., 1961.

ANDERSON, Emily (Hrsg.). The Letters of Mozart and his Family. London: Palgrave Macmillan, 1989.

ANDREOLI, Carlo. Scelta sistematica e progressiva delle opere di Clementi. Milano: Ricordi, 1878.

APPLETON, Elizabeth. Private Education, or A Practical Plan for the Studies of Young Ladies. London: Henry Colburn, 1816.

ARCHENHOLZ, Johann Wilhelm von. A Picture of England. Dublin: P. Byrne, 1791.

BACHARACH, Alfred Louis - PEARCE, John R. (Hrsg.). The musical companion. San Diego - New York - London: Harcourt Brace Jovanovich, 1984.

BAILLIE, Laureen. The catalogue of printed music in the British Library to 1980. London: K. G. Saur, 1982.

BROUGHTON, Vernon Delves (Hrsg.). Court and Private Life in the Time of Queen Charlotte: Being the Journals of Mrs Papendiek, Assistant Keeper of the Wardrobe and Reader to Her Majesty, 2 Bd. London, 1887.

BURGH, Allatson. Anecdotes of Music, Historical and Biographical, in a Series of Letters from a Gentleman to his Daughter. London: Longman, Hurst, Rees, Orme and Brown, 1814.

BURNEY, Charles. A General History of Music from the Earliest Ages to the Present Period (1766-1784). London: G. T. Foulis, 1935.

BUSBY, Thomas. A Dictionary of Music, Theoretical and Practical. To which is prefixed a Familiar Introduction to the First Principles of that Science. London: Sir Charles Phillips \& Co., 1823.

CESI, Beniamino. Metodo per lo studio del pianoforte. Milano: Ricordi, 1897.

CLEMENTI, Muzio. 25 studi estratti dal Gradus ad Parnassum. Milano: Lucca, 1871.

CLEMENTI, Muzio. Gradus ad Parnassum, 34 studi per pianoforte scelti ed ordinati secondo i programmi uniformi di Licenza e Magistero stabiliti dal Ministero della I.P. per gli Istituti Musicali del Regno. Hrsg. Frugatta. Milano: Ricordi, 1898.

CLEMENTI, Muzio. Gradus ad Parnassum, 40 studi scelti. Hrsg. Sgambati. Milano: Ricordi, 1916.

CLEMENTI, Muzio. Per le classi di Pianoforte. R. Istituto musicale di Firenze. 50 studi estratti dal Gradus ad Parnassum da far seguito agli studi di Cramer editi da Hans von Bülow. Progressivamente ordinati e provveduti di nuove diteggiature annotazioni e di segni per la esecuzione dei medesimi da Giuseppe Buonamici. Firenze: Genesio Venturini, 1894.

COLMAN, George. The Musical Lady. A Farce. London: T. Becket und P. A. Dehondt, 1762.

DIRUTA, Girolamo. Il Transilvano. Dialogo sopra il vero modo di sonar organi et istromenti da penna. Venezia: [s. n.], 1593, anastatischer Druck, Bologna: Forni, 1997.

DURKHEIM, Émile. Rappresentazioni individuali e rappresentazioni collettive. In Le regole del metodo sociologico. Milano: Comunità, 1979.

FENAROLI, Fedele. Regole musicali per i principianti di cembalo. Napoli: [s. n.], 1775.

GELLRICH, Martin. Üben mit Lis(z)t. Wiederentdeckte Geheimnisse aus der Werkstatt der Klaviervirtuosen. Frauenfeld: Waldgut, 1992.

GRATTAN FLOOD, William Henry. John Field of Dublin, Inventor of the Nocturne. Dublin: Martin Lester, 1921.

HABERMAS, Jürgen. Storia e critica dell'opinione pubblica. Bari: Laterza, 1971.

HOFMEISTER, Adolph. Handbuch der musikalischen Literatur, oder allgemein systematisch geordnetes Verzeichnis der in Deutschland und in den angrenzenden Ländern erschienenen Musikalien, bearbeitet und herausgegeben von Adolph Hofmeister, 5. Band oder 2. Ergänzungsband die von Anfang 1860 bis 
Ende 1867 neu erschienenen und neu aufgelegten musikalischen Werke enthaltend. Leipzig: [s. n.], 1868 .

HOFMEISTER, Adolph. Handbuch der musikalischen Literatur, oder allgemein systematisch geordnetes Verzeichnis der im deutschen Reiche und in den angrenzenden Ländern erschienenen Musikalien, 7. Band oder 4. Ergänzungsband die von Anfang 1868 bis Ende 1873 neu erschienenen und neu aufgelegten musikalischen Werke enthaltend. Leipzig: [s. n.], 1876.

HOFMEISTER, Adolph. Handbuch der musikalischen Literatur, oder allgemein systematisch geordnetes Verzeichnis der im deutschen Reiche und in den angrenzenden Ländern erschienenen Musikalien, 8. Band oder 5. Ergänzungsband die von Anfang 1874 bis Ende 1879 neu erschienenen und neu aufgelegten musikalischen Werke enthaltend. Leipzig: [s. n.], 1881.

HOFMEISTER, Adolph. Handbuch der musikalischen Literatur, oder allgemein systematisch geordnetes Verzeichnis der im deutschen Reiche und in den angrenzenden Ländern erschienenen Musikalien, 9. Band oder 6. Ergänzungsband die von Anfang 1880 bis Ende 1885 neu erschienenen und neu aufgelegten musikalischen Werke enthaltend. Leipzig: [s. n.], 1887.

HOFMEISTER, Adolph. Handbuch der musikalischen Literatur, oder allgemein systematisch geordnetes Verzeichniß der im deutschen Reiche und in den angrenzenden Ländern erschienenen Musikalien, 10. Band oder 7. Ergänzungsband die von Anfang 1886 bis Ende 1891 neu erschienenen und neu aufgelegten musikalischen Werke enthaltend. Leipzig: [s. n.], 1893.

HOFMEISTER, Adolph. Handbuch der musikalischen Literatur, oder allgemein systematisch geordnetes Verzeichniß der im deutschen Reiche, in den Ländern deutschen Sprachgebietes sowie der für den Vertrieb im deutschen Reiche wichtigen, im Auslande erschienenen Musikalien, 14. Band oder 11. Ergänzungsband die von Anfang 1909 bis Ende 1913 neu erschienenen und neu aufgelegten musikalischen Werke enthaltend. Leipzig: [s. n.], ohne Jahresangabe.

HOFMEISTER, Adolph. Handbuch der musikalischen Literatur, oder allgemein systematisch geordnetes Verzeichnis der im deutschen Reiche, in den Ländern deutschen Sprachgebietes sowie der für den Vertrieb im deutschen Reiche wichtigen, im Auslande erschienenen Musikalien, 11. Band oder 8. Ergänzungsband die von Anfang 1892 bis Ende 1897 neu erschienenen und neu aufgelegten musikalischen Werke enthaltend. Leipzig: [s. n.], 1900.

HOFMEISTER, Adolph. Handbuch der musikalischen Literatur, oder allgemein systematisch geordnetes Verzeichniß der im deutschen Reiche, in den Ländern deutschen Sprachgebietes sowie der für den Vertrieb im deutschen Reiche wichtigen, im Auslande erschienenen Musikalien, 13. Band oder 10. Ergänzungsband die von Anfang 1904 bis Ende 1908 neu erschienenen und neu aufgelegten musikalischen Werke enthaltend. Leipzig: [s. n.], ohne Jahresangabe.

HOHL TRILLINI, Regula. The Gaze of the Listener. English Representations of Domestic Music-Making. Amsterdam - New York: Rodopi, 2008.

HORKHEIMER, Max - ADORNO, Theodor Wiesengrund. Dialettica dell'illuminismo. Torino: Einaudi, 1966.

HUMPHRIES, Charles - SMITH, William C. Music Publishing in the British Isles, from the Beginning until the Middle Part of the Nineteenth Century. New York: Barnes \& Noble, 1970.

KALKBRENNER, Friedrich Wilhelm. Méthode pour apprendre le piano-forte à l'aide du guide-mains. Paris: Meissonnier Fils, 1830.

KENTNER, Louis. Piano. New York: Schirmer, 1976.

LIMONGELLI, Silvia. La didattica per principianti e dilettanti. In RATTALINO, Piero (Hrsg.). Trecento anni, li dimostra? Il pianoforte nella società di oggi. Como: Tipografia Nani Editrice, 2000, S. $123-128$.

MARANO, Maria. Muzio Clementi e la fondazione della didattica pianistica. Cosenza: Luigi Pellegrini, 2007. 
MARISI, Rossella. Musica e divulgazione culturale. München - Ravensburg: Grin, 2009.

MILTON, John. Paradise Lost. Hrsg. Gordon Teskey. London - New York: Norton, 2005.

MISTRETTA, Pietro (Hrsg.). L'insegnamento musicale in Italia. Firenze: Le Monnier, 1986.

MOSCHELES, Charlotte. Life of Moscheles, 2 Bd. Boston: Adamant Media Corporation, 2001.

PADULA, Alessandra. Forma come contenuto, 2 Bd. Torino: Giacomelli, 2002.

PADULA, Alessandra. L'interpretazione come percorso di conoscenza: confronti, problemi, prospettive. München - Ravensburg: Grin, 2007.

PADULA, Alessandra. Le chiavi della musica. Torino: Giacomelli, 1999.

PADULA, Alessandra. Musica e pedagogia tra rigore scientifico e creatività artistica. La forma dello Studio. In PERIGOZZO, Enrico (Hrsg.). Il Quaderno del Conservatorio, n. 3. [S. 1.]: Conservatorio di Musica di Pescara, 2005, S. 33-35.

PADULA, Alessandra. Virtuosismo e virtuosi. In PERIGOZZO, Enrico (Hrsg.). Il Quaderno del Conservatorio, n. 2. [S. 1.]: Conservatorio di Musica di Pescara, 2004, S. 69-71.

PARK, Robert E. - BURGESS, Ernest W. - MCKENZIE, Roderisk D. La città. Milano: Comunità, 1967.

PARSONS, Talcott. Il sistema sociale. Milano: Comunità, 1951.

PERIGOZZO, Enrico (Hrsg.). Il Quaderno del Conservatorio, n. 2. [S. 1.]: Conservatorio di Musica di Pescara, 2004.

PERIGOZZO, Enrico (Hrsg.). Il Quaderno del Conservatorio, n. 3. [S. 1.]: Conservatorio di Musica di Pescara, 2005.

PETTY, F. C. Italian opera in London: musicians and the King's Theatre in London, 1760-1800. Dissertation. Yale: [s. n.], 1971.

PLANTINGA, Leon. Clementi, Virtuosity, and the German Manner. Journal of the American Musicological Society, XXV, 1972, S. 323-329.

PLANTINGA, Leon. Clementi: his life and music. London - New York - Toronto: Oxford University Press, 1977.

RELLSTAB, Ludwig. Ludwig Berger, ein Denkmal. Berlin: [s. n.], 1846.

Reminiscences of Samuel Wesley, British Museum, Add. MS. 27593, 147.

ROSENGREN, Karl E. (Hrsg.). Effects and Beyond: Culture, Socialisation and Lifestyles. London: Routledge, 1994.

SCHINDLER, Anton Felix. Beethoven as I knew Him. A Biography. Hrsg. D.W. Macardle. Chapel Hill: University of North Carolina Press, 1966.

UNGER, Max. Muzio Clementis Leben. Langensalza: Hermann Beyer und Söhne, 1914.

WEBER, Max. I fondamenti razionali e sociologici della musica. In WEBER, Max. Economia e società. Milano: Edizioni di Comunità, 1961, Anhang zu Bd. 2.

WEBER, Max. L'etica protestante e lo spirito del capitalismo. Firenze: Sansoni, 1970.

WOllenberG, Susan - MCVEIGH, Simon (Hrsg.). Concert Life in Eighteenth-century Britain. Aldershot - Burlington: Ashgate, 2004. 